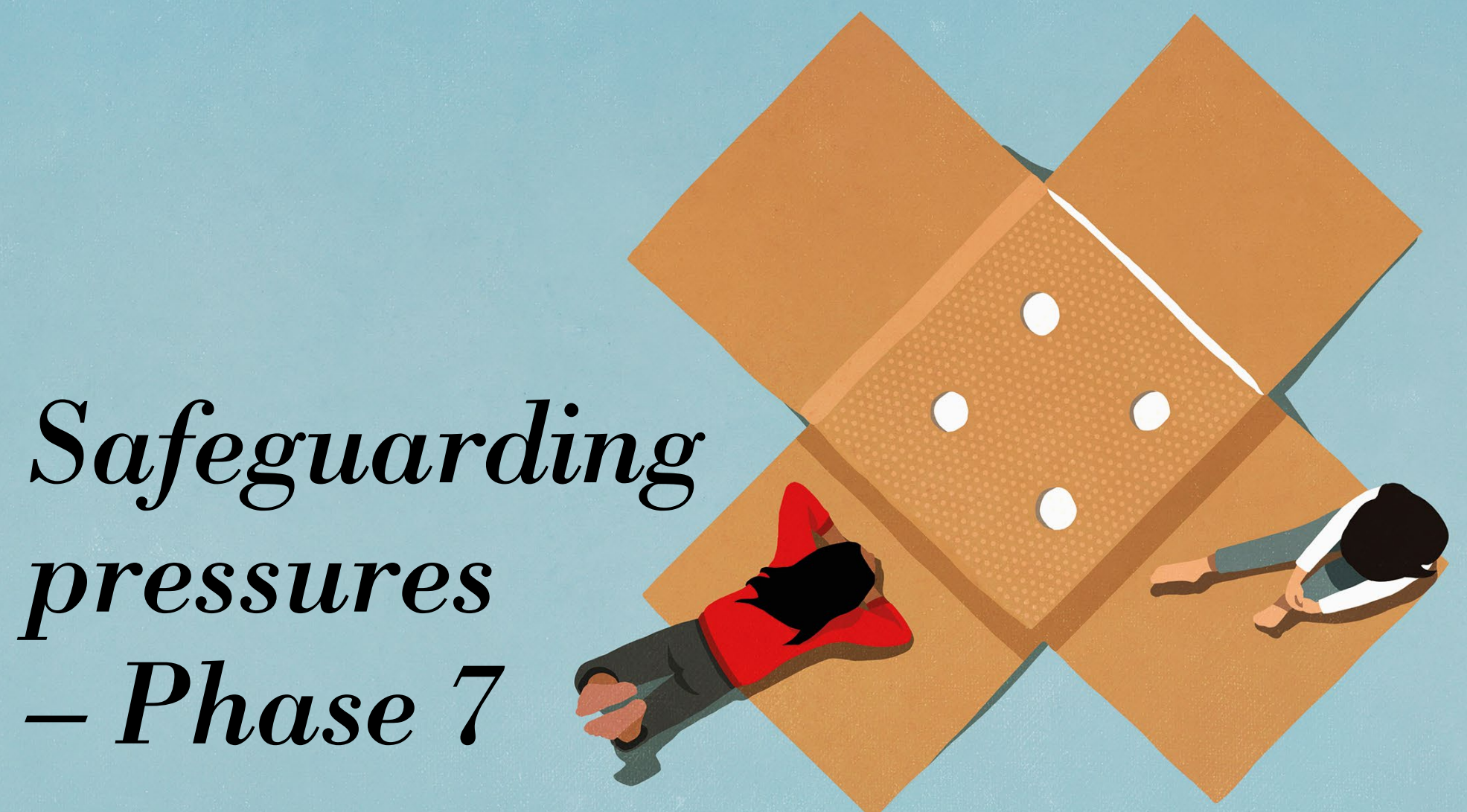

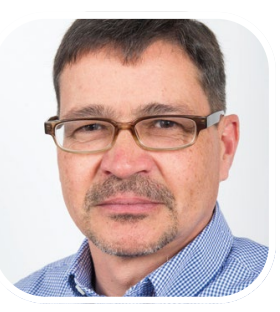

Mark Foster of the Child Protection Company outlines the key points in a new report on safeguarding pressures faced by local authorities, and why dental professionals should stay informed.

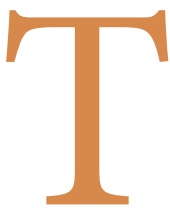

he latest report of the Association of Directors of Children's Services (ADCS) 'Safeguarding Pressures Phase $7^{\prime 1}$ - outlines pressures faced by local authorities during 2019/20 while also including a focus on activity in the first six months of the COVID-19 pandemic. At 127 pages it's a weighty tome but it does help you to understand the current landscape and the pressures children and their parents that you come into contact with, may be under.

For this report ADCS collected evidence from 129 local authorities, covering $89 \%$ of England's children and young people population.

\section{Safeguarding during the COVID-19 pandemic}

In the initial six months of the COVID-19 pandemic, more children were referred who were not previously known to social care services. Families who were just about managing pre-pandemic and would not normally come to the attention of social care were now in need of significant help. Local authorities reported an increase in children becoming subjects of child protection; more children remained subjects of plans and fewer children were stepped down from plans due to potential heightened risk, and absence of other support services.

Adults experiencing domestic abuse, mental health difficulties or substance misuse, remain the most common reasons why children come to the attention of early help and/or children's social care services. A range of studies report the prevalence and impact of these factors on children's safety and wellbeing.

Domestic abuse was cited as the most prevalent and is a prominent factor in re-referral and repeat child protection plans, showing just how difficult it is to achieve sustainable change in circumstances where domestic abuse is present.

\section{Child population in England}

There were 12 million children aged $0-17$ in England in 2019, 156,611 more than two years earlier. The $0-17$ age population is projected to increase to 12.2 million in 2025 .

Poverty

In 2018/19 there were 2.7 million children and young people living in relative low income. The continued impact of welfare reforms, families affected by the benefit cap, in-work poverty, and the economic downturn were significant determinants of presenting factors such as, for example, parental mental ill health, domestic abuse, parental substance misuse and child neglect.

The impact of the COVID-19 pandemic was reported by respondents to be extremely 
concerning in terms of exacerbating disadvantage for children and their families.

Social care services and early help Police continue to be the biggest source of contacts and referrals, with the number (and proportion of the total) from health and education increasing incrementally over the years. Primary health services and A\&E departments are the two biggest components of health referrals. Indeed, referrals from A\&E alone outstrip those from school nurses, health visitors and GPs combined.

For every 10,000 children ( 0 to 17 years) in England in 2019/20 there were:

- 2,098 Initial contacts

- 535 Referrals

- 423 Section 47 enquiries (where there are reasonable grounds to suspect that a child is suffering or is likely to suffer significant harm)

- 202 Early help assessments

- 167 Children in Need (including Child Protection and Child Looked After)

- 67 Children Looked After

- 42.8 Child protection plans

- 36 care leavers age 17-21 (a Care Leaver is someone who has been in the care of the local authority for a period of 13 weeks or more spanning their 16th birthday)

- 4 Unaccompanied Asylum-Seeking Children (UASC).

The most common sources of contacts and referrals have changed since March 2020. There were fewer referrals from schools, due to school closures, and an increase in those from the public and self-referrals.

The child's behaviour, parenting, domestic abuse and the child's learning or physical disability or illness are the most prevalent needs, or reasons for involvement, cited in early help assessments.

Parental domestic abuse, parental mental health and emotional abuse continue to be the most prevalent factors in social care assessment, with significant increases in these factors over the past two years.

Whilst multiple needs can be recorded for early help, this is not the case in statutory reporting of referrals, child protection plans, and children looked after plans. There is an increase in the proportion of referrals which have a primary need of abuse and/or neglect to $62.4 \%$ of all referrals in 2019/20.

More children are subjects of child protection plans for neglect and emotional abuse, and fewer for physical or sexual abuse. More children are starting to be looked after due to Abuse or Neglect, an increase to $62.5 \%$ of all children looked after in 2019/20.

Children and young people requiring support from children's services are presenting with more complex and multiple needs. Whilst this has been increasingly evident over the past two years, it has been more acute during the pandemic and is forecast to increase further as the full impact of the pandemic is realised. or aware of what actions are needed to stem the spiralling factors that contribute to poor outcomes for children, young people and families.

\section{So how does this affect me?}

Basically, it's important you report any safeguarding concerns you may have about any child or vulnerable adult you come into contact with. It may be vital in preventing

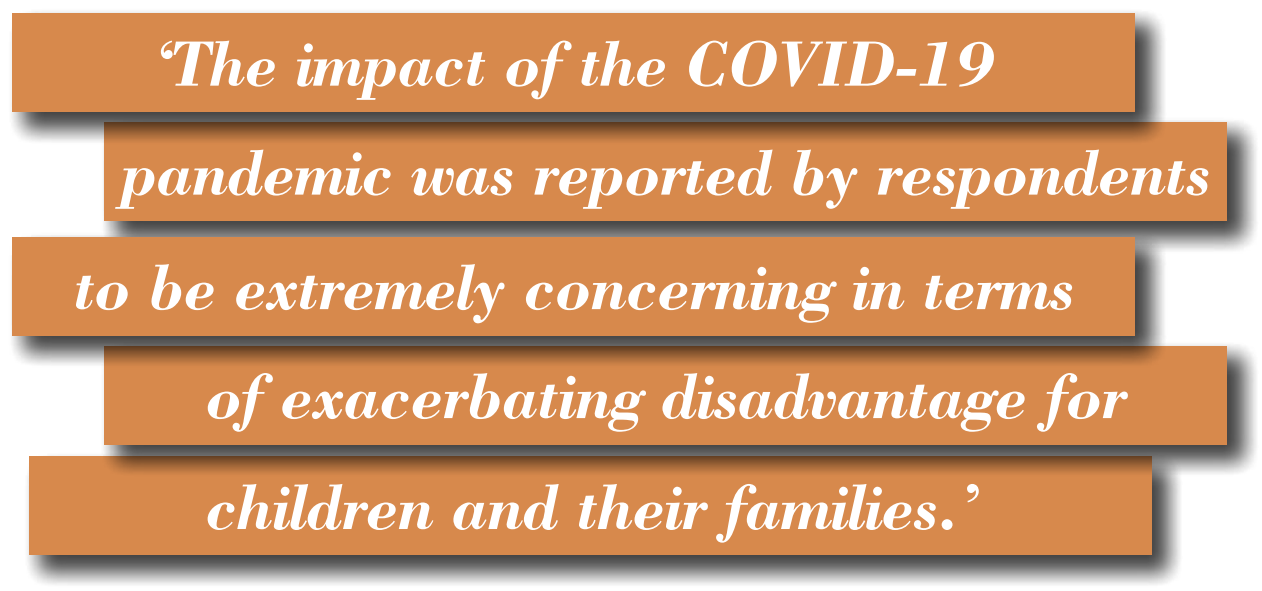

\section{Conclusion}

Over the 12 years covered by ADCS's research, local authorities have evidenced $87 \%$ more initial contacts, $19 \%$ more referrals, $3 \%$ more children in need, $76 \%$ more children being the subjects of child protection plans and $32 \%$ more children looked after.

The longer term and lasting impact of the COVID-19 pandemic on families remains to be seen however, with respondents identifying 'latent need' that is yet unknown. As the pandemic eases, local authorities anticipate significant increases in children's emotional and mental health needs.

Moreover, the wider societal determinants of family distress including employment, housing concerns and poverty will emerge as pressures that plunge families that would ordinarily manage into crisis.

Key changes predicted include an increase in poverty and worklessness; domestic abuse; parental and child mental health difficulties; parental substance misuse; potential antisocial behaviour and community unrest; a widening of the gap for disadvantaged children; the long-term impact for children experiencing hidden harms; an upward trajectory of admissions to hospital and care due to deteriorating mental health and the negative impact of school closures on educational outcomes.

However, there is evidence that children's services leaders are implementing, influencing abuse occurring or reoccurring. In line with your practice's procedures report any concerns you have to your practice Safeguarding Lead. You will not be criticised for raising your concerns.

If you're the practice's Safeguarding Lead then where a referral is required, it should be made within 24 hours. Also, it's important to make sure everyone's training is up to date. If they're not, the Child Protection Company offers BDA recommended online dental specific safeguarding courses which can be accessed immediately. Visit https://www. childprotectioncompany.com/CPC/p/dental/.

Remember, a child and vulnerable adult's safety and welfare is paramount and the sharing of information with the relevant individuals, organisations and agencies is absolutely essential.

Safeguarding is everyone's responsibility.

\section{Reference}

1. The Association of Directors of Children's Services Ltd. Research Report. Safeguarding pressures Phase 7. February 2021. Available at: https://adcs. org.uk/assets/documentation/ADCS_ Safeguarding_Pressures_Phase7_FINAL. pdf (accessed November 2021). 